\title{
Influencia del tipo de material, superficie de contacto y altura de almacenaje sobre el ángulo de reposo, en materiales granulares
}

\author{
Influence of type of material, contact surface and storage height on the angle of \\ repose in granular materials
}

${ }^{1}$ Priscila Maldonado Uría

ORCID: 0000-0002-1832-4797

${ }^{2}$ Edwin Pino Vargas

ORCID: 0000-0001-7432-4364

\section{RESUMEN}

En el presente trabajo se buscó analizar la influencia de tres factores principales sobre el ángulo de reposo, los cuales fueron: el tipo de material, combinaciones de arenas de distintos tamaños, rugosidad de la superficie de contacto, superficies de vidrio y lija, y altura de almacenaje del ensayo, de $8 \mathrm{~cm}, 18 \mathrm{~cm}$ y $28 \mathrm{~cm}$. Al interactuar todos los factores estudiados mediante el método del embudo, se determinó el ángulo de reposo. Para corroborar la efectividad del método del embudo, partiendo de la simplificación: ángulo de fricción crítico $(\varphi c)=$ ángulo de reposo ( $\varphi r e p)$, se realizó el ensayo de corte directo calculando así el ángulo de fricción crítico para cada tipo de material y compararlo con el ángulo de reposo, los cuales tuvieron resultados similares.

Palabras clave: Ángulo de fricción crítico ( $\varphi c)$, ángulo de reposo ( $\varphi r e p)$, corte directo, método del embudo.

\begin{abstract}
In the present work we analized the influence of three main factors on the angle of repose, which were: the type of material, combinations of sand of different sizes, roughness of the contact surface, glass and sandpaper surfaces, and height of test storage, heights of $8.18,28 \mathrm{~cm}$. By interacting all the factors by the funnel method, the angle of repose was determined. To corroborate the effectiveness of the funnel method, based on the simplification critic friction angle $(\varphi c)=$ angle of repose ( $\varphi$ rep), the direct shear test was performed calculating the critic friction angle for each type of material and comparing it with the angle of repose, which had similar results.
\end{abstract}

Keywords: Critical friction angle $(\varphi c)$, angle of repose ( $\varphi r e p)$, direct shear, funnel method.

${ }^{1}$ Compañía de minas Buenaventura S.A.A. Área de Geología/exploraciones. E-mail: p.maldonadou23@gmail.com

${ }^{2}$ Universidad Nacional Jorge Basadre Grohmann. Escuela de Ingeniería Civil. E-mail: epino68@hotmail.com 


\section{INTRODUCCIÓN}

Actualmente, en el Perú no existe ninguna metodología consolidada para obtener el ángulo de reposo de los suelos granulares ni estudios sobre los factores que influyen sobre este y el impacto que producen.

El ángulo de reposo es fundamental en la estimación de los valores máximos de inclinación de masas de suelos granulares, como taludes de suelos granulares y terraplenes de carreteras. Las variaciones que se puedan producir en los valores del ángulo de reposo, se traducen en variaciones en los factores de seguridad y en importantes diferencias de volúmenes de suelo a excavar; por lo tanto, tiene un impacto directo tanto en la seguridad como en los costos de los proyectos.

Asimismo, el ángulo de fricción crítico $(\varphi c)$ es uno de los parámetros de mayor evaluación e importancia para la determinación de la estabilidad de taludes, presas de relave, botaderos, resistencia de cimentaciones superficiales y profundas, en presiones laterales, en el diseño y construcción de carreteras y en los cálculos de empujes de tierra.

En materiales granulares, el ángulo de fricción crítico está relacionado al ángulo de reposo. Pudiendo obtener el ángulo de fricción crítico $(\varphi c)$ desde ensayos de compresión triaxial o por corte directo simple. Por otro, lado el ángulo de reposo ( $\varphi r e p)$ se puede obtener por distintos métodos, siendo uno de los más utilizados el método del embudo, el cual consiste en la construcción de una pila por medio de un embudo. Según Solaque y Lizcano (2008), se puede considerar al ángulo de fricción crítico igual que el ángulo de reposo ( $\varphi c=\varphi r e p)$, se utiliza esta simplificación por la similitud de los resultados obtenidos para estos dos ángulos (Herle y Gudehus, 1999).

El presente trabajo de investigación busca aportar información de manera práctica y experimental sobre el ángulo de reposo, buscando determinar la influencia del tipo de material y la rugosidad de la superficie de contacto sobre el ángulo de reposo en función a las alturas de almacenaje variables. Asimismo, comparar el ángulo de reposo con el ángulo de fricción crítico, para la comprobación de la efectividad del método del embudo para el cálculo de este parámetro.
Considerando el aspecto académico, proporciona una visión de cómo distintos factores tienen influencia sobre el parámetro estudiado. Asimismo, proporciona información de referencia para trabajos de investigación similares que tienden a la aplicación en proyectos de obras geotécnicas.

\section{MATERIAL Y MÉTODOS}

\section{Conformación de materiales}

Del trabajo de campo, se extrajeron aproximadamente $200 \mathrm{~kg}$ de suelo fino (pasante malla $\mathrm{N}^{\circ} 4$ ). Del material recolectado en campo, se tamizó la arena por tamaño de partícula, para conformar los cuatro tipos de materiales a ensayar con una específica granulometría. Los tipos de materiales ensayados se conformaron de la siguiente forma: El tipo 1 es de $100 \%$ de arena gruesa; el tipo 2 es de $60 \%$ de arena gruesa y $40 \%$ de arena media; el tipo 3 es de $40 \%$ de arena gruesa, $40 \%$ de arena media y $20 \%$ de arena fina; y el tipo 4 es de $25 \%$ de arena gruesa, $35 \%$ de arena media, $35 \%$ de arena fina y $5 \%$ de limos.

\section{Ensayos de laboratorio}

Para controlar la composición de todos materiales ensayados, se realizaron ensayos de granulometría, utilizando los tamices $\mathrm{N}^{\circ} 4, \mathrm{~N}^{\circ} 10$, $\mathrm{N}^{\circ} 20, \mathrm{~N}^{\circ} 40, \mathrm{~N}^{\circ} 60, \mathrm{~N}^{\circ} 100 \mathrm{y} \mathrm{N}^{\circ} 200$ (norma ASTM D422), para corroborar la invariabilidad de la composición de los materiales luego de cada ensayo del embudo.

También, se realizó el ensayo de límites de Atterberg (norma ASTM D4318) para el material tipo 4 , esto para confirmar que se trabajara con limos.

Para un control de la humedad de cada ensayo del embudo, se realizaron ensayos de contenido de humedad (norma ASTM D2216) en cada uno.

Para conocer la densidad o gravedad específica de cada tipo de material, se realizó el ensayo de gravedad específica (norma ASTM D854).

Para el cálculo del ángulo de fricción crítico, con el fin de compararlo con los resultados obtenidos por el método del embudo para el ángulo de reposo, se realizó el ensayo de corte directo (norma ASTMD3080) para cada tipo de material. 


\section{Método del embudo}

Existen varios métodos utilizados para calcular el ángulo de reposo de un material (Rodas y Rouse, 2010). Entre estos, el más usado es el método del embudo; este busca determinar el ángulo de reposo de los materiales ensayados. Se debe garantizar que el diámetro de salida del embudo sea mayor o igual a cinco veces el diámetro de la partícula (Dembudo $>5$ dpartícula) (Gundogdu et al., 2008). Asimismo, hay diversas formas de medir el ángulo de reposo, una es tratar de medir el ángulo del cono resultante estimando, directamente, el valor gráficamente; la otra forma es dividir el doble de la altura por el diámetro de la base del cono. La tangente inversa de esta relación es el ángulo de reposo.

Según el método I, desarrollado por Train (1958), el embudo empleado en la conformación de la pila se mantiene a una altura constante $\mathrm{H}$ y el material se vierte dentro del embudo para que la pila quede perfectamente cónica. La pila se construye hasta que toque el orificio de salida del embudo. Luego, se mide el diámetro de la base de la pila (D) y se determina el ángulo de reposo de la siguiente forma. Ecuación (1).

$\varphi_{\text {rep }}=\arctan \left(\frac{2 h}{\text { Dprom }}\right)$

Donde:

$h \quad:$ Altura del cono $(\mathrm{cm})$

Dprom: Diámetro promedio del cono $(\mathrm{cm})$

$\varphi_{\text {rep }} \quad:$ Ángulo de reposo $\left({ }^{\circ}\right)$

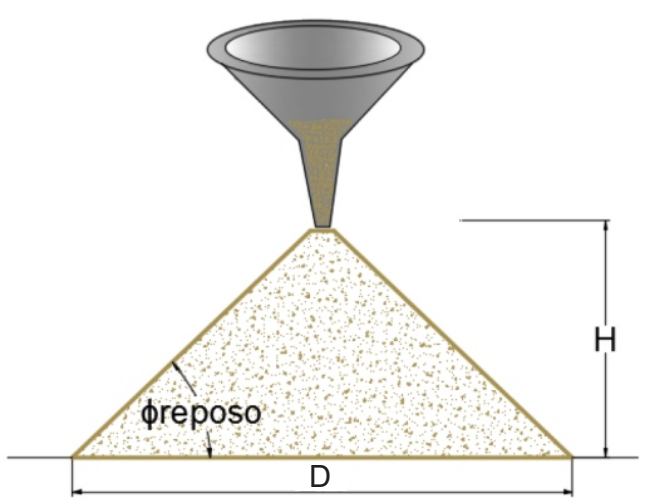

Figura 1. Método descrito por Train para determinar el ángulo de reposo (Barrantes y León, 2017).

\section{RESULTADOS}

\section{Ensayos de laboratorio}

Granulometría

Se realizó el análisis granulométrico para cada tipo de material, secando el material antes de ensayarlo.

Tabla 1. Valores granulométricos de los materiales ensayados

\begin{tabular}{llllll} 
Tipo & $\begin{array}{l}\mathbf{D}_{\mathbf{1 0}} \\
(\mathbf{m m})\end{array}$ & $\begin{array}{l}\mathbf{D}_{\mathbf{3 0}} \\
(\mathbf{m m})\end{array}$ & $\begin{array}{l}\mathbf{D}_{\mathbf{6 0}} \\
(\mathbf{m m})\end{array}$ & $\mathbf{C}_{\mathbf{U}}$ & $\mathbf{C}_{\mathbf{C}}$ \\
\hline 1 & 1.48 & 1.77 & 1.95 & 1.32 & 1.08 \\
2 & 0.51 & 0.78 & 1.51 & 2.97 & 0.79 \\
3 & 0.16 & 0.34 & 0.93 & 5.92 & 0.80 \\
4 & 0.11 & 0.16 & 0.40 & 3.80 & 0.5
\end{tabular}

Se realizó el análisis granulométrico al inicio y termino del ensayo del embudo y se corroboró que no se da ningún cambio significativo en la composición del material ensayado.

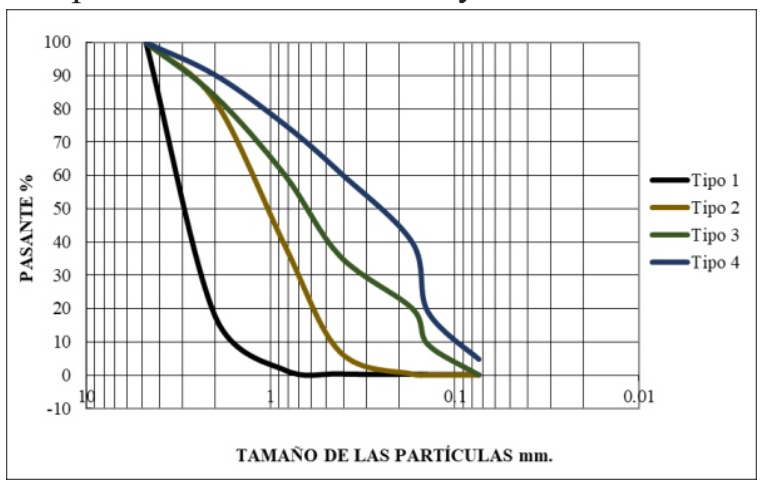

Figura 2. Comparación de curvas granulométricas por tipo de material

\section{Límites de consistencia}

El material tipo 4 ensayado presentó un límite líquido de $17.43 \%$, límite plástico de $0 \%$ y no mostró índice de plasticidad (NP). Según la clasificación SUCS, basado en la norma ASTM D2487-00 (2000), las partículas finas del material tipo 4 correspondieron a limos.

\section{Clasificación de suelos}

Con los datos del análisis granulométrico y límites de consistencia de los cuatro tipos de materiales ensayados, se los clasificó mediante el sistema SUCS, perteneciendo todos a arena mal graduada (SP), basada en la norma ASTM D 2487-00 (2000). 


\section{Contenido de humedad}

De acuerdo a los análisis de contenido de humedad de los materiales ensayados, se obtuvieron los siguientes resultados: tipo 1 con $\omega$ $=0.33 \%$, tipo $2 \omega=0.95 \%$, tipo $3 \omega=1.02 \%$, tipo $4 \omega=0.83 \%$.

\section{Gravedad específica}

Los valores obtenidos son muy similares en todos los casos, ya que cada tipo de material fue constituido por la misma materia prima, pero en diferentes porcentajes cada uno. Para el tipo 1 de $2.67 \mathrm{~g} / \mathrm{cm}^{3}$, tipo $2 \mathrm{de} 2.65 \mathrm{~g} / \mathrm{cm}^{3}$, tipo 3 de 2.67 $\mathrm{g} / \mathrm{cm}^{3}$ y tipo $4 \mathrm{de} 2.69 \mathrm{~g} / \mathrm{cm}^{3}$.

\section{Ángulo de reposo}

Para determinar el ángulo de reposo, se utilizó el método del embudo, ensayado para cada tipo de material, utilizando las muestras correspondientes a las combinaciones con 2 tipos de superficie: lisa - vidrio y rugosa - lija. Asimismo, trabajando con 3 alturas constantes en cada caso h1 $(8 \mathrm{~cm}), \mathrm{h} 2(18 \mathrm{~cm})$ y h3 $(28 \mathrm{~cm})$, realizando 3 ensayos por cada muestra, para obtener datos representativos.

Tabla 2. Valores del ángulo de reposo, interactuando superficie, altura y tipo de material

\begin{tabular}{|c|c|c|c|c|c|c|}
\hline \multirow[t]{2}{*}{ 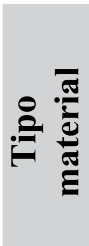 } & \multicolumn{2}{|c|}{$\begin{array}{c}\mathrm{H1} \\
(\mathbf{8} \mathrm{cm})\end{array}$} & \multicolumn{2}{|c|}{$\begin{array}{c}\mathrm{H} 2 \\
(18 \mathrm{~cm})\end{array}$} & \multicolumn{2}{|c|}{$\begin{array}{c}\mathrm{H3} \\
(28 \mathrm{~cm})\end{array}$} \\
\hline & Lisa & Rug. & Lisa & Rug. & Lisa & Rug \\
\hline \multirow{2}{*}{ T1 } & 32.5 & 31.7 & 34.1 & 34.1 & 339 & 34.0 \\
\hline & 2 & 1 & & 1 & 7 & \\
\hline \multirow{2}{*}{$T_{2}^{2}$} & 31.3 & 31.9 & 32.2 & 34.2 & 32.6 & 33.1 \\
\hline & 6 & 6 & & 4 & 5 & 5 \\
\hline & 30.7 & 32.3 & 32.3 & 32.3 & 33.1 & 32.8 \\
\hline & 0 & 6 & & 0 & 5 & 4 \\
\hline & 30. & 31. & 33.9 & 34. & 33.8 & 33.9 \\
\hline & 8 & 3 & 6 & 1 & 7 & 3 \\
\hline
\end{tabular}

En la Tabla 2 se resumen los valores del ángulo de reposo, obtenidos realizando las combinaciones entre los tipos de materiales con alturas ensayadas sobre las superficies de contacto. Se obtuvieron valores similares, teniendo un valor máximo de $34.24^{\circ} \mathrm{y}$ un valor mínimo de $30.70^{\circ}$, teniendo entonces una máxima variación de $3.54^{\circ}$.

\section{$\underline{\text { Corte Directo }}$}

Se realizaron los ensayos de corte directo, en condiciones secas, para los cuatro tipos de materiales ensayados, a diferentes esfuerzos normales. Los parámetros de resistencia resultantes de cada tipo de material, se muestran en la Tabla 3.

Tabla 3. Parámetros de resistencia de los materiales ensayados

\begin{tabular}{cccccc}
$\begin{array}{c}\text { Tipo de } \\
\text { Material }\end{array}$ & \multicolumn{2}{c}{$\mathbf{C}\left(\mathbf{K g f} / \mathbf{c m}^{2}\right)$} & \multicolumn{2}{c}{$\Phi^{\circ}$} \\
\hline 1 & 0.092 & 0.060 & 33.66 & 31.59 \\
2 & 0.084 & 0.042 & 32.86 & 30.92 \\
3 & 0.095 & 0.024 & 33.46 & 29.42 \\
4 & 0.086 & 0.041 & 29.98 & 26.20 \\
\end{tabular}

Los ángulos de fricción críticos o residuales $\varphi c$ se determinaron mediante ensayos de corte directo, con saturación $0 \%$. Estos se realizaron con los mismos materiales empleados en los ensayos del ángulo de reposo, con el fin de compararlos, considerando $\varphi c=\varphi r e p$, planteado por Soloque (2008).

\section{A N ÁL IS IS DE FACTORES DE INFLUENCIA}

Cabe mencionar que la presente investigación no contó con la capacidad de poder materializar una pila de material granular grueso a escala real, distintas superficies de contacto y de alturas de almacenaje controladas, para poder analizar las influencias de estos factores.

Es por esto que se realizan ensayos en materiales granulares con granulometrías correspondientes a arenas y finos, que son representativos para los estudios correspondientes al presente trabajo de investigación.

\section{Influencia del tipo de material}

Parte del objetivo general del presente trabajo fue determinar la influencia del tipo de material sobre el ángulo de reposo. Para esto, se conformaron cuatro tipos de materiales compuestos por arena gruesa, media, fina y finos, en porcentajes descritos anteriormente; estos porcentajes se 
eligieron para obtener las combinaciones necesarias para el análisis de las variaciones.

En la Tabla 4 se muestran los resultados promedios del ángulo de reposo obtenido en los cuatro tipos de material ensayados, mostrando su composición y clasificación SUCS. Este análisis se realizó con el fin de determinar si existía variación sobre el ángulo de reposo, por composición de material.

Tabla 4. Composición de material en relación a su ángulo de reposo calculado

\begin{tabular}{|clcc|}
$\begin{array}{c}\text { Tipo de } \\
\text { material }\end{array}$ & Composición & $\begin{array}{c}\text { Clasificación } \\
\text { SUCS }\end{array}$ & $\begin{array}{c}\boldsymbol{\Phi}^{\mathbf{o}} \text { rep } \\
\text { promedio }\end{array}$ \\
\hline $1 \quad \begin{array}{l}100 \% \text { Arena } \\
\text { gruesa }\end{array}$ & SP & $33.42^{\circ}$ \\
\hline 2 & $\begin{array}{l}60 \% \text { A. gruesa, } \\
40 \% \text { a. media }\end{array}$ & SP & $32.61^{\circ}$ \\
& $\begin{array}{l}40 \% \text { A. gruesa, } \\
40 \% \text { a. media, }\end{array}$ & SP & $32.28^{\circ}$ \\
& $\begin{array}{l}20 \% \text { a. fina } \\
25 \% \text { A. gruesa, }\end{array}$ & & \\
4 & $\begin{array}{l}35 \% \text { a. media, } \\
35 \% \text { a. fina, } 5 \%\end{array}$ & SP & $32.96^{\circ}$ \\
& Finos
\end{tabular}

Como se observa en la Figura 8, en el caso del material tipo 1 compuesto únicamente por arena gruesa se obtuvo un ángulo de reposo de $33.4^{\circ}$, siendo el mayor valor obtenido. Para los tipos 2 y 3 , los resultados son similares, oscilando en $32^{\circ}$, esto se debe a que los materiales correspondieron a mezclas de arenas con el mismo tamaño máximo y mínimo de partículas, por ende, presentan el mismo relleno de vacíos entre partículas. Cabe mencionar que al mismo tiempo se observa una ligera tendencia de disminución del valor del ángulo de reposo a medida que disminuye la granulometría.

Para el material tipo 4, se evidencia un ligero aumento en el valor de ángulo de reposo, rompiendo la tendencia de disminución que se evidenciaba en el tipo 2 y 3 . Esto se debe a que este material incorpora en su composición un pequeño porcentaje de limos, los que generan una ligera cohesión y ligero aumento del relleno de vacíos entre partículas, resultando en el incremento del valor del ángulo de reposo respecto al tipo 2 y 3 .

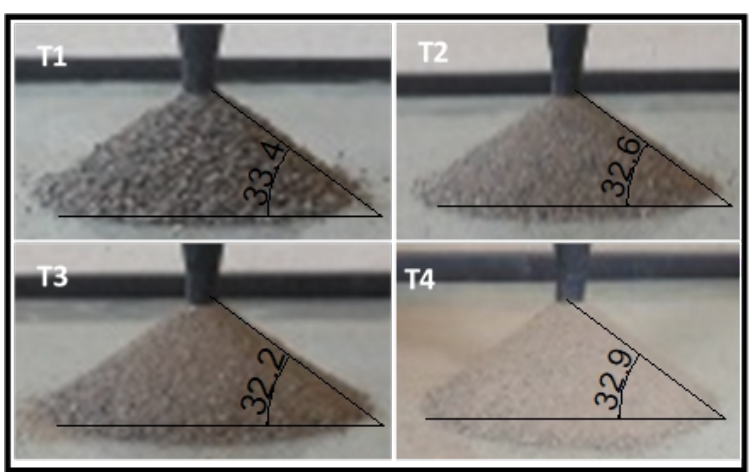

Figura 3. Ángulo de reposo por tipo de material

Como se explicó líneas arriba, los resultados presentan una tendencia al decrecimiento del ángulo de reposo ( $\varphi r e p)$ a medida que la granulometría disminuye.

Estos resultados coinciden con el análisis presentado por Solaque \& Lizcano (2008), así también con Herle \& Gudehus (1999), quienes concluyen en su investigación: "Al rodar las partículas en el proceso de formación de la pila, los granos grandes tienden a superar esfuerzos más altos. Esto puede explicar un incremento de prep al aumentar el tamaño de los granos".

Pero a su vez difieren cuando el material presenta un porcentaje de limos en su composición, explicando este comportamiento líneas arriba.

\section{Influencia de la rugosidad de las superficies de contacto}

En este trabajo se realizaron todos los ensayos sobre dos superficies de contacto, una de vidrio y la otra de lija gruesa con rugosidades lisa y rugosa respectivamente, esto para poder analizar la influencia de cada una sobre los resultados del ángulo de reposo. En la Tabla 5 se observan los resultados obtenidos.

Tabla 5. Ángulo de reposo en relación a las superficies por tipo de material

\begin{tabular}{|cccc|}
$\begin{array}{c}\text { Tipo de } \\
\text { material }\end{array}$ & $\begin{array}{c}\text { Superficie } \\
\text { lisa } \\
\boldsymbol{\Phi}^{\mathbf{0}} \text { rep }\end{array}$ & $\begin{array}{c}\text { Superficie } \\
\text { rugosa } \\
\boldsymbol{\Phi}^{\mathbf{0}}{ }_{\text {rep }}\end{array}$ & $\begin{array}{c}\text { Variación } \\
\mathbf{( ~}^{\mathbf{0}} \mathbf{)}\end{array}$ \\
\hline 1 & 33.55 & 33.29 & 0.26 \\
2 & 32.10 & 33.12 & 1.02 \\
3 & 32.06 & 32.50 & 0.44 \\
4 & 32.90 & 33.02 & 0.12 \\
\hline
\end{tabular}

Los ensayos mencionados se realizaron primero en una superficie de vidrio, esto para no generar ningún tipo de fuerza, debido a la fricción entre el 
material granular y la base durante el proceso de formación de la pila.

De la misma manera, se realizaron también los mismos ensayos sobre una superficie de lija gruesa, la cual genera una pequeña fuerza de fricción entre el material y la superficie de contacto.

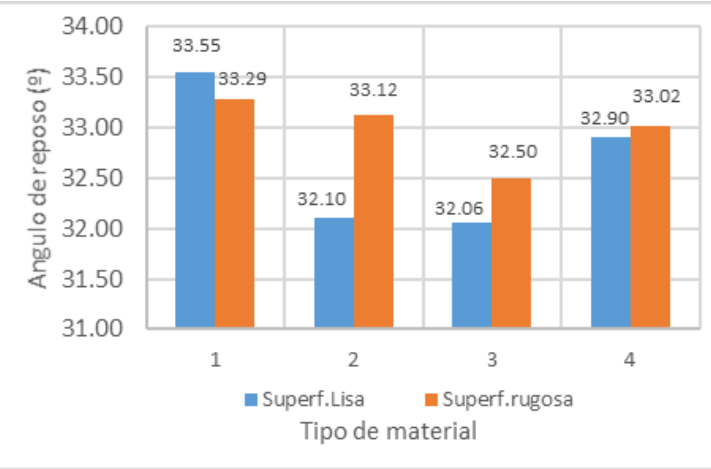

Figura 4. Comparación del ángulo de reposo por tipo de material ensayado en cada superficie

Como se observa en la Figura 4, la máxima variación obtenida ensayando el mismo tipo de material fue de $1.02^{\circ}$, pudiéndose observar también que en la superficie rugosa se obtuvieron los valores de ángulo de reposo ligeramente más altos. Se puede deducir de esto que, la rugosidad de la superficie de contacto del ensayo produce una ligera variación sobre el valor del ángulo de reposo de un material.

Este factor de rugosidad fue estudiado por Chik y Vallejo (2005), quienes concluyeron: "La rugosidad de la base afecta más el ángulo de reposo para partículas gruesas. Cuando el porcentaje de finos es mayor o igual al $70 \%$ de la masa de la mezcla (predomina fracción fina), la rugosidad de la base no influye".

Con base en estos resultados bibliográficos y a los resultados del presenta trabajo de investigación se coincide que para materiales finos la rugosidad no es un factor de importante influencia sobre el ángulo de reposo.

Cabe mencionar que se evidencia que la rugosidad de la superficie influencia en la cantidad de masa necesaria para conformar la pila; ya que, en una superficie de vidrio al no presentar una fuerza de fricción, el material resbala, lo que genera mayor masa para conformar la pila; en cambio en una superficie rugosa donde la superficie de contacto genera una ligera fuerza de oposición, el material no resbala fácilmente.

Al construir las pilas cónicas de material sobre una superficie lisa (vidrio) y una rugosa (lija gruesa), se encontró que el tipo de superficie de contacto afecta ligeramente a la forma de los taludes de las pilas de material. Para la superficie rugosa se obtiene una pila convexa (Figura 10b) bien constituida.

Sobre la superficie de vidrio, la forma de la pila es convexa, pero a su vez el talud presenta una parte curva en su base (Figura 10a), ya que, al ir formándose la pila en la base de vidrio, los bordes de esta ceden al caer el material mientras se van acomodando las partículas.

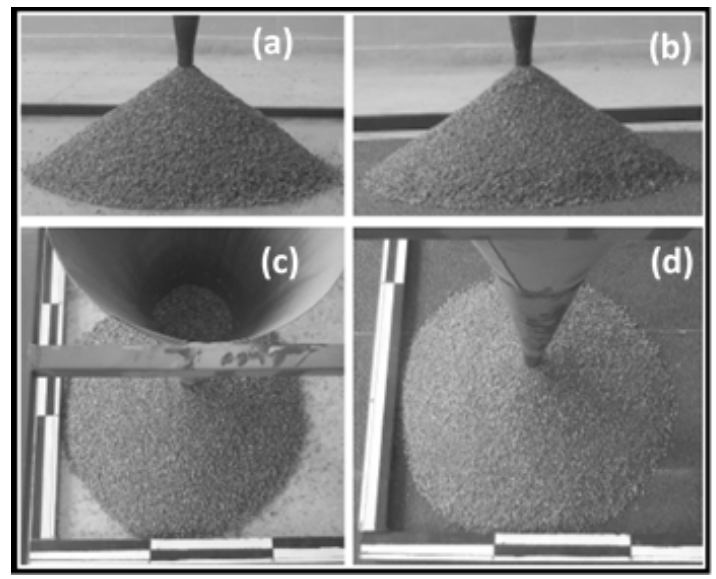

Figura 5. Comparación de la forma de la pila cónica de material T1, en superficie lisa y rugosa.

\section{Influencia de la altura}

Se realizaron los mismos ensayos para cada muestra a tres alturas fijas de h1 de $8 \mathrm{~cm}, \mathrm{~h} 2$ de 18 $\mathrm{cm} \mathrm{y} \mathrm{h3}$ de $28 \mathrm{~cm}$ (Figura 6). Los resultados obtenidos para cada tipo de muestra en las tres alturas mencionadas, se observan en la Tabla 6.

Tabla 6. Ángulo de reposo en relación a la altura en cada tipo de material

\begin{tabular}{|cccc|}
$\begin{array}{c}\text { Tipo de } \\
\text { material }\end{array}$ & $\begin{array}{c}\text { H1 } \\
\mathbf{( 8 c m )}\end{array}$ & $\begin{array}{c}\mathbf{\Phi}_{\text {rep }}^{\mathbf{~}} \\
\mathbf{( 1 8 c m )}\end{array}$ & $\begin{array}{c}\mathbf{H 3} \\
\mathbf{( 2 8 c m )}\end{array}$ \\
\hline T1 & 32.12 & 34.14 & 34.02 \\
T2 & 31.66 & 33.26 & 32.90 \\
T3 & 31.53 & 32.31 & 33.00 \\
T4 & 30.96 & 34.04 & 33.90 \\
\hline
\end{tabular}




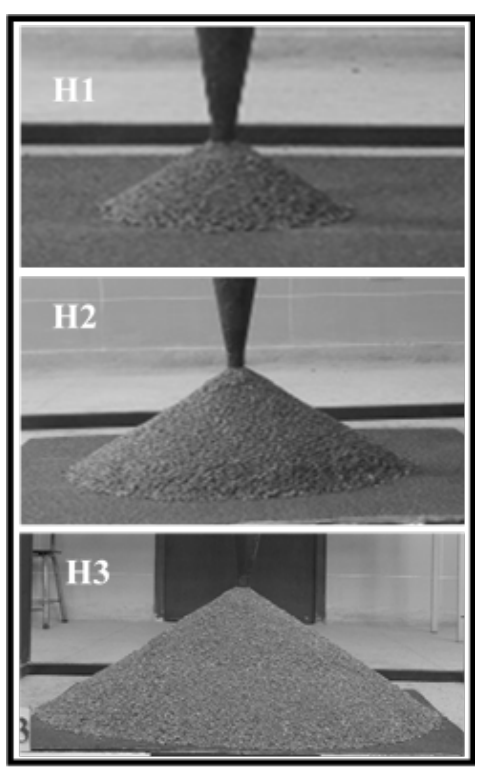

Figura 6. Pilas cónicas del material T1 con superficie rugosa, mostrando las tres alturas.

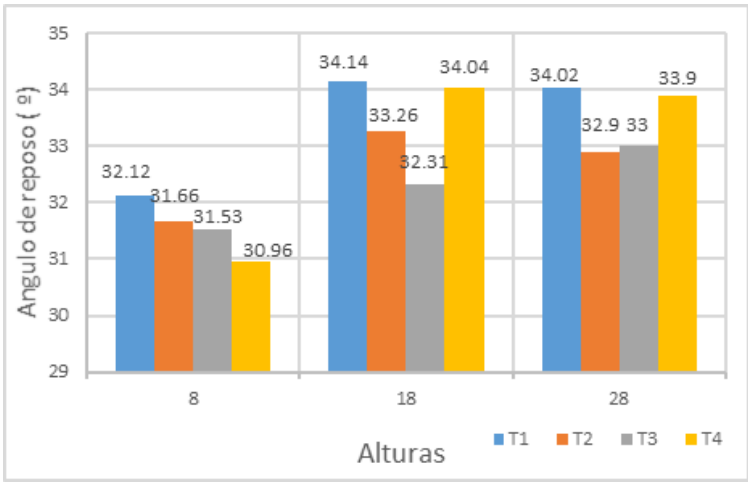

Figura 7. Valores de ángulo de reposo para cada tipo de material en tres alturas.

En la Figura 7 se puede observar que los menores valores del ángulo de reposo corresponden a una altura inicial h1 de $8 \mathrm{~cm}$.

Por otro lado, los resultados obtenidos para h2 y h3 aumentan respecto a los obtenidos en $\mathrm{h} 1$, pero ambos son similares. Se observa que, al aumentar la altura del ensayo de $18 \mathrm{~cm}$ a los $28 \mathrm{~cm}$, en promedio, el ángulo de reposo aumenta su valor en menor proporción, teniendo resultados similares, para un h2 de $33.44^{\circ}$ y para h3 de $33.46^{\circ}$.

Se realizó también el análisis respecto a la masa utilizada dependiendo del tipo de material y la altura del ensayo. Pudiendo observar en la Tabla 7 que, al aumentar la altura de ensayo, la masa requerida en promedio se cuadruplica.

Se observa también que la mayor cantidad de masa utilizada se encuentra en el material 3 y 4 , pudiendo deducir de esto que al aumentar la cantidad de finos (\% de arenas fina y finos) se necesita mayor masa para ocupar el mismo volumen.

Tabla 7. Masa utilizada en relación a la altura en cada tipo de material

\begin{tabular}{cccc|}
$\begin{array}{c}\text { Tipo de } \\
\text { material }\end{array}$ & $\begin{array}{c}\text { H1 } \\
\mathbf{( 8 c m )}\end{array}$ & $\begin{array}{c}\text { H2 } \\
\mathbf{( 1 8 c m )}\end{array}$ & $\begin{array}{c}\text { H3 } \\
\mathbf{( 2 8 c m})\end{array}$ \\
\hline T1 & 4.000 & 23 & 82.000 \\
T2 & 4.410 & 26.060 & 87.000 \\
T3 & 4.800 & 26.890 & 88.000 \\
T4 & 4.820 & 26.000 & 84.000 \\
\hline
\end{tabular}

\section{ANÁLISIS DE INTERRELACIONES}

\section{Comparación del ángulo de reposo con el ángulo de fricción interna}

Se realizó el análisis comparativo entre el ángulo de fricción crítico calculado mediante el ensayo de corte directo para los cuatro tipos de materiales, y el ángulo de reposo calculado mediante el ensayo del método del embudo en los cuatro tipos de materiales ensayados interactuando con los dos tipos de rugosidad y tres alturas; partiendo de la igualdad ángulo de fricción crítico $(\varphi c)$ igual al ángulo de reposo ( $\varphi r e p)$ (Soloque, 2008). Se mostraron los resultados de ambos ángulos obtenidos para el mismo tipo de material en la Tabla 8.

Tabla 8. Comparación entre el ángulo de fricción crítico y ángulo de reposo

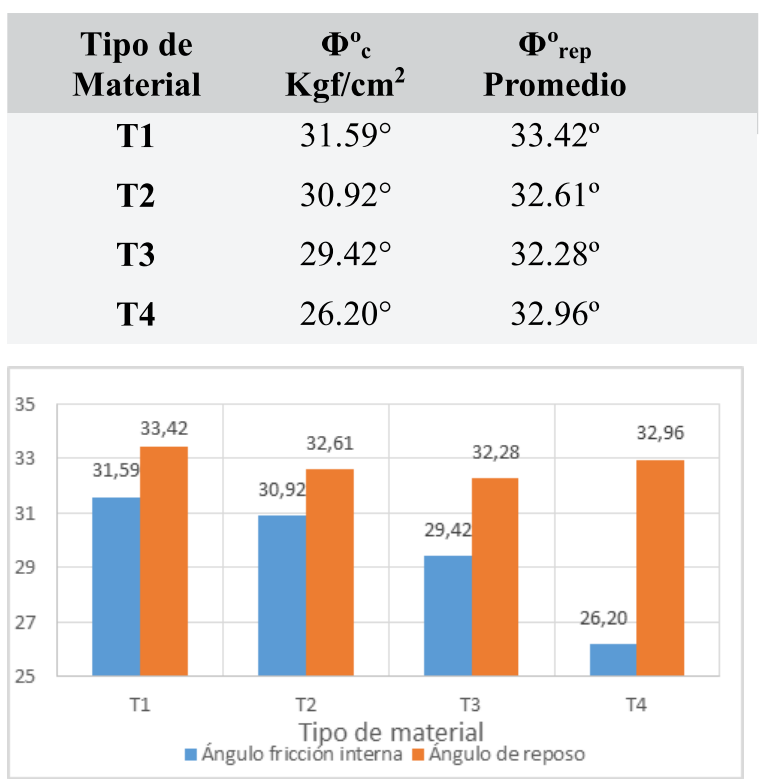

Figura 8. Comparación del ángulo de fricción interna y el ángulo de reposo promedio, por tipo de material. En la Figura 8 se observa que la mayor para los 
valores del ángulo de fricción crítico y ángulo de reposo se presenta en los materiales de tipo 3 y 4 , con mayores porcentajes de arenas medias, finas y limos.

Para comprobar que, para materiales granulares estos dos ángulos pueden ser considerados similares, se aplicó la prueba estadística "T" de Student, que evalúa la diferencia aceptable de dos resultados. En la Tabla 9 se presentan los resultados, obteniendo un valor estadístico "t" 2.77 , menor al valor crítico de $t$ de 3.18 , con estos resultados podemos dar como correcta la hipótesis de proximidad de los valores de los ángulos de reposo y de fricción crítico.

Tabla 9. Prueba “ $T$ ” para la diferencia de medias entre el ángulo de reposo y de fricción crítico

$\begin{array}{lcc} & \begin{array}{c}\text { Ángulo } \\ \text { reposo }\end{array} & \begin{array}{c}\text { fricción } \\ \text { crítico }\end{array} \\ \text { Media } & 32.82 & 29.53 \\ \text { Varianza } & 0.24 & 5.76 \\ \text { Observaciones } & 4.00 & 4.00 \\ \text { Coeficiente de correlación } & 0.15 & \\ \text { de Pearson } & & \\ \text { Diferencia hipotética de las } & 0.00 \\ \text { medias } & & \\ \text { Grados de libertad } & 3.00 \\ \text { Estadístico t } & 2.77 \\ \text { P(T<=t) una cola } & 0.03 \\ \text { Valor crítico de } \mathrm{t} \text { (una cola) } & 2.35 \\ \text { P(T<=t) dos colas } & 0.07 \\ \text { Valor crítico de t (dos colas) } & 3.18\end{array}$

\section{Análisis de comportamientos y patrones}

Con los resultados obtenidos se analizó el comportamiento que presenta el ángulo de reposo respecto al ángulo de fricción interno. Para esto se realizó el análisis de los promedios y luego el análisis de tendencia por tipo de superficie y altura.

\section{Análisis con valores promedios}

De cada tipo de material ensayado se ploteó la data del ángulo de reposo promedio de las 24 muestras, calculado en cada una de las combinaciones con tipo de superficie y altura utilizada, eligiendo valores promedios para cada tipo de materiales y comparándolos con los valores del ángulo de fricción crítico obtenidos del ensayo de corte directo, para los mismos tipos de materiales.

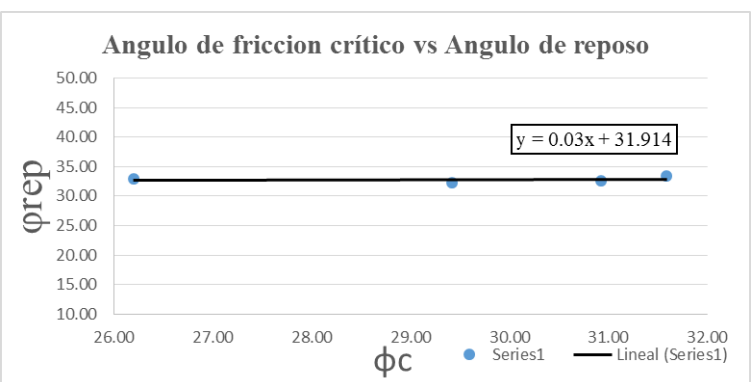

Figura 9. Ángulo de reposo en función del ángulo de fricción interno, construido con los valores promedios de todos los tipos de materiales.

Los resultados muestran que los ángulos de reposo y de fricción interna pueden ser correlacionados mediante una expresión lineal (Figura 9).

De este análisis se concluye que el ángulo de reposo de arenas en estado seco no consolidado es similar al ángulo de fricción interna en estado seco no consolidado. Estos resultados coinciden con los presentados por Ghazavi, Hosseini y Mollanouri (2008), quienes concluyeron que en arenas estos dos ángulos se correlacionan, en condiciones similares de densidad y consolidación, mediante una expresión empírica lineal.

\section{Análisis con valores promedio por tipo de superficie}

Para este análisis se tomaron los promedios de los resultados de las muestras ensayadas sobre superficies lisa y rugosa.

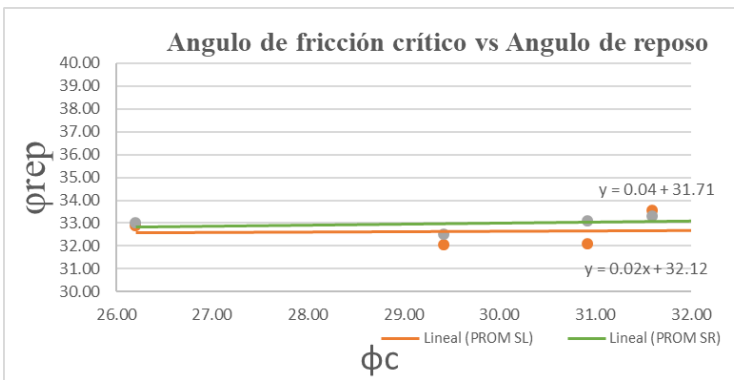

Figura 10. Ángulo de reposo en función del ángulo de fricción interna construido con los valores promedios de todos los tipos de materiales sobre una superficie lisa y rugosa.

Los resultados muestran que el ángulo de reposo y de fricción crítico ensayado sobre una superficie lisa y rugosa pueden ser correlacionados mediante una expresión lineal diferenciándose entre ambas superficies en las décimas de las constantes de las ecuaciones empíricas de cada una (Figura 10). 
De este análisis se observa que el ángulo de reposo es similar al ángulo de fricción crítico en arenas en estado seco no consolidado, concluyendo al igual que Chik y Vallejo (2005) que la rugosidad, en la escala de este estudio, no es un factor influyente en los valores de estos ángulos en materiales granulares finos.

\section{Análisis con valores promedio por altura de ensayo}

Para este análisis se tomaron los promedios de todos los resultados obtenidos en cada tipo de material ensayados a 3 alturas fijas h1, h2 y h3, tomando la data del ángulo de reposo calculado y comparándolo con los valores obtenidos del ensayo de corte directo, para los mismos tipos de materiales.

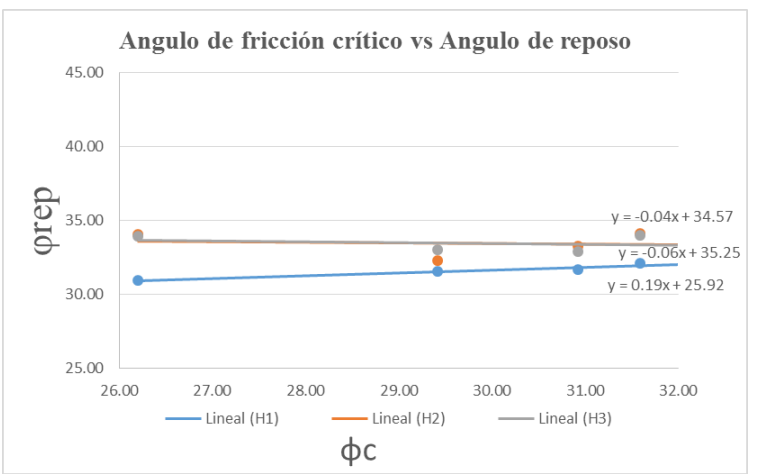

Figura 11. Ángulo de reposo en función del ángulo de fricción interna construido con los valores promedios de todos los tipos de materiales ensayados a tres alturas (h1, h2, h3) fijas.

Mediante una expresión empírica lineal, se correlacionó al ángulo de reposo y al ángulo de fricción interna, concluyendo que la ecuación correspondiente a la correlación de los datos ensayados con altura $\mathrm{H} 1$ presentan una constante menor, acortándose la diferencia entre estos dos ángulos (Figura 11).

\section{CONCLUSIONES}

Los tipos de materiales elegidos para la presente investigación se definieron como arenas mal graduadas (SP), que presentaron $0.33 \% \leq \omega \leq 1.02$ $\%, 2.65 \mathrm{gr} / \mathrm{cm}^{3} \leq G s \leq 2.69 \mathrm{gr} / \mathrm{cm}^{3}$ y el tipo 4 presente un LL $=17.43^{\circ}, \mathrm{IP}=\mathrm{NP}$. Conformando estos cuatro tipos de materiales, cuya composición buscó la combinación de todos los tipos de arenas según tamaño de partícula: arenas gruesas, medias y finas en diferentes porcentajes; considerando para el tipo 4 un $5 \%$ de limos.
Se eligieron dos tipos de superficie para evaluar si la rugosidad de la base es un factor influyente sobre el ángulo de reposo de cada material. Una superficie lisa de vidrio para que no ejerza ninguna fuerza de fricción y una superficie rugosa de lija $\mathrm{N}^{\circ} 80$ con coeficiente de fricción de 0.32 , según bibliografía.

Se establecieron tres alturas fijas para los ensayos, las cuales fueron de $8 \mathrm{~cm}, 18 \mathrm{~cm}$ y $28 \mathrm{~cm}$. Estas se eligieron para poder evaluar el impacto del ensayo trabajando con alturas que varían en aumento de $10 \mathrm{~cm}$ de una a la otra.

De los resultados de los ensayos con todas las combinaciones de material, superficie y altura, con un total de 24 muestras, se obtuvo que el ángulo de reposo calculado por el método del embudo, y el ángulo de fricción crítico calculado por corte directo presentaron valores de $30.70^{\circ} \leq \phi$ reposo $\leq$ $34.24^{\circ}$, ensayados en estado seco, mediante el ensayo de corte directo en condiciones secas 26.20 ${ }^{\circ} \leq \phi \mathrm{c} \leq 31.59^{\circ}$.

Pudiendo ser correlacionados mediante una expresión lineal con una constante promedio de $3.15^{\circ}$, en estado seco no consolidado (UU), concluyendo que estos dos ángulos son similares y la vez se muestra que el ángulo de reposo es ligeramente mayor que el ángulo de fricción crítico, obteniendo en promedio una diferencia de $3.29^{\circ}$.

En cuanto a la influencia que ejerce el tipo de material sobre el ángulo de reposo, se concluye que, al trabajar con arenas el ángulo decrece ligeramente a medida que el tamaño de partículas disminuye.

El análisis de la rugosidad de la superficie del ensayo mostró que el ángulo de reposo en una superficie rugosa aumenta ligeramente respecto a los resultados sobre una superficie lisa. Al ser esta variación máxima de $1.02^{\circ}$, se concluye que, a la escala del estudio, este no es un factor que influya de manera significativa.

En cuanto al análisis de la influencia de la altura del ensayo, la mayor diferencia en cuanto al ángulo de reposo se da al trabajar con una altura de $8 \mathrm{~cm}$, teniendo una variación de $1.9^{\circ}$, en comparación con los resultados obtenidos para alturas de $18 \mathrm{~cm}$ y $28 \mathrm{~cm}$. Concluyendo que el factor altura mostró una ligera influencia creciente y constante al aumentar la altura. 


\section{REFERENCIAS}

ASTM International. ASTM D2216. 1998. Obtenido de Método de prueba estándar para la determinación en laboratorio del contenido de agua (humedad) de suelos y rocas por masa. Disponible en: https://www.astm.org/DATABASE.CA RT/HISTORICAL/D2487-00.htm

ASTM International. ASTM D422. 1998. Obtenido de Método de análisis del tamaño de las partículas de suelos: https://www.astm.org/DATABASE.CA RT/HISTORICAL/D2487-00.htm

ASTM International. ASTM D2487. 2000. Obtenido de Standard Classification of Soils for Engineering Purposes (Unified Soil Classification System): https://www.astm.org/DATABASE.CA RT/HISTORICAL/D2487-00.htm

ASTM International. ASTM D3080. 2011. Obtenido de Standard Test Method for Direct Shear Test of Soils Under Consolidated Drained Conditions: https://www.astm.org/Standards/D3080 .htm

ASTM International. ASTM D 854. 2014. Obtenido de métodos de prueba estándar para la gravedad específica de sólidos del suelo por agua picnómetro: h t t p : / / w w w a s t m.org / c g i bin/resolver.cgi?D854-14

ASTM International. ASTM D 4318. 2017. Obtenido de Standard Test Methods for Liquid Limit, Plastic Limit, and Plasticity Index of Soils: https://www.astm.org/Standards/D4318 .htm

BARRANTES, S., \& LEÓN, A. Ángulo de reposo en suelos granulares de Lambayeque. Tesis de pregrado. Universidad Señor de Sipán. Trujillo, Perú. 2017.

CHIK, Z., \& VALLEJO, L. Characterization of the angle of repose of binary granular materials. Canadian Geotechmical Journal 42(2): 683-692, 2005.
G H A Z AV I, M ., H O S S E IN I, M . , \& MOLLANOURI, M. A Comparison between Angle of Repose and Friction Angle of Sand. 12th International Conference of the 12th Internacional Association for Computer Methods and Advences in Geomechanics (IACMAG), 1272- 1275, 2008.

GUNDOGDU, M. Discharge Characteristics of Polydisperse Powders through Conical Hoppers. Part 2: Predictions for Coarse, Granular. Free Flowing Powders. Particulate Science and Technology, 2337, 2006.

GUNDOGDU, M., KUTLAR, A., \& CARPINLIOGLU, M. Discharge Regimes of Polydisperse Powders through Conical Hoppers under Positive Flow Conditions. Particulate Science and Technology, 551-562, 2008.

HERLE, I., Y GUDEHUS, G. Determination of Parameters of a Hypoplastic Constitutive Model from Properties of Grain Assemblies. Mechanics of Cohesive-Frictional Materials, 4(5): 461-486, 1999.

RODAS, R., y ROUSE, P. Análisis comparativo de métodos para la medición del ángulo de reposo de suelos granulares. Revista de la Construccion 9(1): 98-106. 2010.

SOLAQUE, D., Y LIZCANO, A. Ángulo de fríccion cíitico y ángulo de reposo de la arena del Guamo. Epsilon, 7-20, 2008.

SOLOQUE, D. Comparación del ángulo de fricción crítico con el ángulo de reposo. Análisis de la influencia de algunos factores en la determinación del ángulo de reposo. Universidad de los Andes. 2008.

TRAIN, D. Some aspects of the property of angle of repose of powders. Journal of Pharmacy and Pharmacology, 10, 127135,1958 . 